\title{
Severe Spastic Trismus without Generalized Spasticity after Unilateral Brain Stem Stroke
}

\author{
Jong-Hyun Seo, M.D., Don-Kyu Kim, M.D., Ph.D., Si Hyun Kang, M.D., \\ Kyung-Mook Seo, M.D., Ju Won Seok, M.D. ${ }^{1}$ \\ Departments of Physical Medicine and Rehabilitation, ${ }^{1}$ Nuclear Medicine, \\ Chung-Ang University College of Medicine, Seoul 156-755, Korea
}

\begin{abstract}
A 62-year-old female patient diagnosed with left brain stem stroke 2 months ago was admitted to our clinic for rehabilitation. She had no generalized spasticity on both extremities, but could open her mouth only approximately $2 \mathrm{~mm}$ between her upper and lower teeth due to severe trismus. On needle electromyography, the left masseter muscle showed paradoxically increased muscle activity during mouth opening. We injected 50 units of type A botulinum toxin $\left(\right.$ Botox $^{\circledR}$ ) into the left masseter muscle, and 20 units into the left temporalis muscle with guidance of ultrasonography. The interincisal distance increased to $8 \mathrm{~mm}$ on the $3^{\text {rd }}$ day after injection, and $9 \mathrm{~mm}$ on the $4^{\text {th }}$ day. One month later, the interincisal distance increased to $14 \mathrm{~mm}$. The increased interincisal distance was maintained for 13 months after injection, and the quality of hygienic care and compliance of oral stimulation therapy also improved.
\end{abstract}

Key Words Trismus, Botulinum toxin, Brain stem stroke

\section{INTRODUCTION}

Trismus from spastic hypertonia of masticatory muscles is an upper motor neuron syndrome that is caused after different types of cerebral damage such as traumatic brain injury, hypoxic brain damage, and stroke. It can cause serious complications after cerebral damages, because mouth opening is essential for oral feeding,

Received January 3, 2011; Accepted April 21, 2011

Corresponding author: Don-Kyu Kim

Department of Physical Medicine and Rehabilitation, Chung-Ang University Hospital, 224-1, Heuk-suk-dong, Dong-jak-gu, Seoul 156-755, Korea

Tel: +82-2-6299-1881, Fax: +82-2-6298-1866, E-mail: donkim21@gmail. com

() This is an open-access article distributed under the terms of the Creative Commons Attribution Non-Commercial License (http:// creativecommons.org/licenses/by-nc/3.0) which permits unrestricted noncommercial use, distribution, and reproduction in any medium, provided the original work is properly cited.

Copyright $\odot 2012$ by Korean Academy of Rehabilitation Medicine oral hygiene, and speech. However, trismus after brain stem lesion is known to be a rare complication, ${ }^{1}$ whereas trismus after stroke in the cerebral hemisphere or severe traumatic brain injury is relatively common. ${ }^{2}$ In these previous reports on trismus after stroke in cerebral hemisphere or severe traumatic brain injury, various treatments were tried such as stretching exercise, massage, and several antispasmodics, and the results were different from case to case. Botulinum toxin, which has been used for hypertonic muscular activity from different etiologies, was used for trismus after cerebral damage with relatively successful results. ${ }^{3-5}$ However, the location of injection, the volume of botulinum toxin injected, the type of bolulinum toxin, and the duration of followup were different in each case. We report a rare case of trismus after unilateral brainstem ischemic stroke, which had paradoxical eletromyographic activity of the masseter muscle and was showing sustained improvement for 13 
months after the injection of type A botulinum toxin.

\section{CASE REPORT}

A 62-year-old female patient had a history of admission to the emergency care center with decreased mental status. She was diagnosed as having acute infarction on the left brain stem following magnetic resonance imaging (MRI). After conservative management including intravenous tissue plasminogen activator, comprehensive rehabilitation programs were initiated as she had incomplete quadriplegia with ataxia especially severe on her left extremities. She also had facial palsy on her left side, dysphagia, and history of aspiration pneumonia. On the $60^{\text {th }}$ day of her onset of infarction, she was transferred to our hospital for further rehabilitation management. The follow-up magnetic resonance imaging (MRI) on the day of admission showed cerebromalacia on the left middle cerebral peduncle (Fig. 1-A), lateral medulla oblongata (Fig. 1-B), and lower cerebellum (Fig. 1-C).

On physical examination, she had clear mental status, but severe dysarthria and left peripheral type facial palsy with decreased pain/temperature sensation on left face (Fig. 2-A). Cranial nerve function test showed spontaneous horizontal nystagmus to the right side (2nd degree) following Alexander's law. She had resting tremor, dysmetria, and ataxia on her extremities, and the sensation on her right extremities was decreased.

She had no generalized spasticity on her extremities (Modified Ashworth Scale was grade 0 in all extremities) with relatively good muscle power; motor power was grade 5 on her right side and grade 4 on her left. She
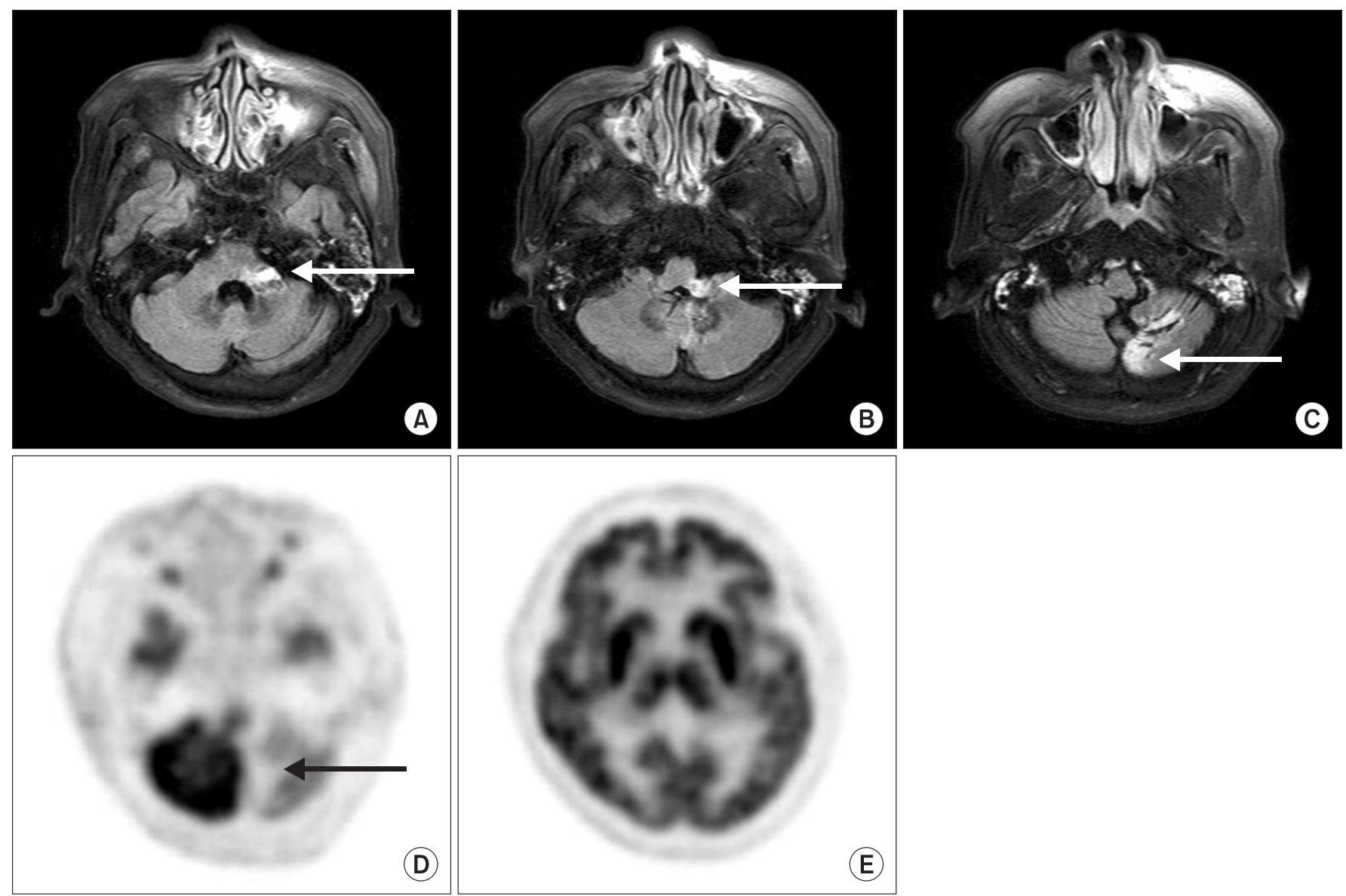

C

Fig. 1. On follow-up magnetic resonance imaging on the day of admission, cerebromalacia on the left middle cerebral peduncle was observed (A), lateral medulla oblongata (B), and lower cerebellum (C). Positron emission tomography (PET) findings were checked to find another brain lesion that can cause the severe trismus. Areas with decreased activities were found on the same areas with abnormalities which were found on MRI such as cerebellum (D), but there were no additional focuses in other areas of the brain $(\mathrm{E})$. 
was unable to open her mouth and the left side opening was more difficult, which was considered to be due to spastic hypertonia of muscles involved in mastication. She could not open her mouth more than $2 \mathrm{~mm}$ in distance between her upper and lower teeth (Fig. 2-B). In addition to dysphagia, oral hygiene was very poor because cleaning or brushing her teeth by a caregiver was impossible. Videofluoroscopic swallowing study was not possible either due to severe trismus. Nasogastric tube for feeding and taking medication had been inserted and tracheostomy tube had been kept for airway maintenance and breathing.

She had no pain and showed no abnormal findings on her temporomandibular joints on physical examination. There were no specific abnormalities on simple x-ray film of both her temporomandibular joints. In addition to magnetic resonance imaging, we checked positron emission tomography (PET) to find another possible brain lesion that could cause the severe trismus, but there were no additional active focuses of abnormalities found by MRI (Fig. 1-D, E). When she tried to open her mouth, her right masseter muscle looked normally relaxed while her left masseter muscle contracted paradoxically, and contracting muscle mass was palpable. On needle electromyography, the left masseter muscle showed increased muscle activity when she tried to open her mouth, as well as when she closed her mouth. From these findings, we speculated the cause of trismus of this patient as paradoxical abnormal spastic hypertonia of the left masseter muscle after brain stem stroke.

Physical modalities such as superficial heat, massage, and stretching exercises were tried without overt improvement of the trismus. We did not choose oral pharmacological regimen because there was no spasticity on her extremities. Thus, under the consideration of the localized spastic dystonia, we injected 50 units of type A botulinum toxin Botox ${ }^{\circledR}$ (Allergan, Irvine, CA) on her left masseter muscle, and 20 units on the left temporalis muscle under the guidance of ultrasonography. We could not inject the toxin into the left medial pterygoid muscle because of limited opening of the mouth. Physical modalities including superficial heat, massage, and stretching exercises were continued and changes in the interincisal distance were serially measured.

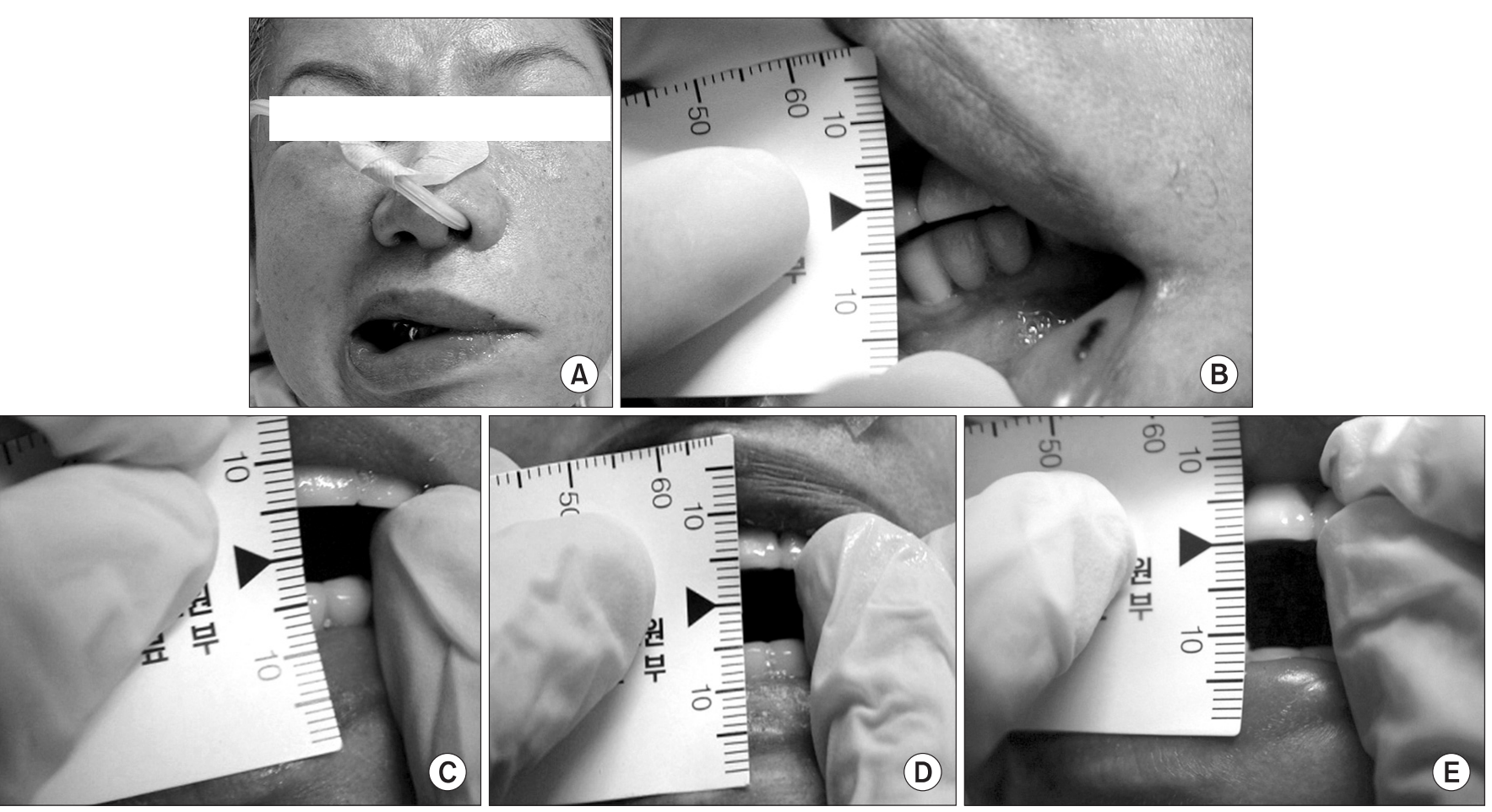

Fig. 2. Before treatment with Botulinum toxin A, the patient had left facial palsy (A), and her interincisal distance was about $2 \mathrm{~mm}$ (B). After treatment, the interincisal distance increased from $2 \mathrm{~mm}$ to $8 \mathrm{~mm}$ on $3^{\text {rd }}$ day after injection (C), and $9 \mathrm{~mm}$ on $4^{\text {th }}$ day (D), and one month after, the interincisal distance increased to $12 \mathrm{~mm}$ and this interincisal distance continued after 3 months of injection (E). 
The interincisal distance increased to $8 \mathrm{~mm}$ on the $3^{\text {rd }}$ day after injection (Fig. 2-C), and $9 \mathrm{~mm}$ on the $4^{\text {th }}$ day (Fig. 2-D). In the seven days following the first injection, injection to the left medial pterygoid muscle was tried but it was still impossible because of the lack of mouth opening.

Videofluoroscopic swallowing study was conducted after she was able to open her mouth about $9 \mathrm{~mm}$ in distance. There was no swallowing reflex and she showed prompt aspiration to the airway when we tested with a small amount $(2 \mathrm{ml})$ of liquid. Because of this, oral feeding was considered impossible. However, the increased interincisal distance improved the quality of hygienic care and made oral stimulation therapy possible. We continued thermal sensory stimulation using ice, oromotor facilitation therapy, tongue exercise, facial massage, and mouth opening training. In one month, the interincisal distance increased to $14 \mathrm{~mm}$ and this interincisal distance was maintained for 3 months after injection (Fig. 2-E). At this point, her Modified Bathel Index improved to 34 points (her Modified Bathel Index on admission was 3 points) due to improvement on transfer and ambulation function as well as on some activities of daily living. However, oral feeding was not possible until discharge to another other hospital and she needed a Levin tube because of sustained dysphagia. Since then, she has been visiting our clinic every other month. When she visited in 13 months after the first injection, she could open her mouth about $14 \mathrm{~mm}$ in distance, but she had a percutaneous endoscopic gastrostomy (PEG) tube for feeding because her dysphagia symptoms did not improve.

\section{DISCUSSION}

After cerebral damage such as stroke or other brain injuries such as trauma, tumor, or Moyamoya disease, trismus may occur and this interferes with oral hygiene, oral feeding, speech, and basic therapy for dysphagia such as oral stimulation and oromotor facilitation. However, trismus after unilateral brain stem lesion described in our case is very rare. Masticatory muscles including masseter, temporalis, and medial pterygoid muscles are all innervated by the $5^{\text {th }}$ cranial nerve (trigeminal nerve). Because of bilateral cortical innervations of the motor trigeminal nucleus, trismus is known to be more common in the bilateral cerebral cortical lesion than in the unilateral lesion. After unilateral mesencephalic lesion, however, paradoxical masticatory muscle hyperactivities from disturbance in co-ordination within mesencephalic nucleus of the $5^{\text {th }}$ nerve could result in trismus. ${ }^{6}$ In our case, MRI showed infarction on the left middle cerebral peduncle, lateral medulla oblongata, and lower cerebellum, which included the mesencephalic nucleus of the trigeminal nerve. We found the increased muscle activities of the left masseter muscle when the patient tried to open her mouth, as well as when she closed her mouth on needle electromyography.

Botulinum toxin has been used to treat hypertonic activities of muscles from various etiologies such as dystonia and cerebral palsy. Recently, several studies have used botulinum toxin for spastic trismus after stroke in the cerebral hemisphere, traumatic brain injury, and hypoxic encephalopathy with relatively successful results. In a placebo-controlled randomized study, they used 2500 units of botulinum toxin type B in each masseter muscle for improvement of trismus after brain damage, and showed significantly increased mouth opening after botulinum toxin injection compared with placebo treatment. ${ }^{4}$ Another report used 75 units of botulinum toxin A to each masseter muscle to improve PEG placement in 18 patients with masseter hyperactivity caused by TBI and ischemic stroke. ${ }^{5}$ In another case report, only 10 units of botulinum toxin A were injected into temporalis muscle with complete resolution of trismus after supratentorial ischemic stroke stroke. ${ }^{3}$ A few studies showed recommended botulinum toxin doses in various diseases such as dystonia, spasticity, and muscle spasm in mandibular muscles. ${ }^{7,8}$ After reviewing these reports, we selected 50 units of bolulinum toxin A into masseter muscle, and 20 units into temporalis muscle according to a previous study. These doses could be changed in various cases with various degrees of spasticity.

After injection of botulinum toxin, the interincisal distance increased which improved the quality of hygienic care and made oral stimulation therapy possible. PEG tube was also successfully placed for feeding. In our case, there were no adverse effects of botulinum toxin such as muscle atrophy or hoarseness. Increased interincisal distance continued for 13 months after the first injection. 
Because of terminal axon sprouting and reinnervation of muscle fibers, reduced hyperactivity after botulinum toxin injection was known to last for 4-6 months after injection. ${ }^{9}$ However, long-lasting effects are also known to be possible. A case study on a pediatric patient with trismus reported the long-term resolution of trismus after repeated injection of botulinum toxin A. ${ }^{10}$ In our study, we observed that the increased interincisal distance lasted for over 1 year. Based on these findings, botulinum toxin A could be safely and successfully used for spastic trismus after brain stem stroke, and methodology of using botulinum toxin A such as volume and repetition of injection should be selected according to the goal of treatment.

We report a rare case of a patient with severe spastic trismus without generalized spasticity after unilateral brain stem stroke. Botulinum toxin A injection for spastic trismus after brain stem stroke improved the patient's oral hygiene, compliance of oral stimulation therapy, and helped PEG tube placement.

\section{REFERENCES}

1. Freitag V, Schulz A. Trismus resulting from central nervous system lesion. J Maxillofac Surg 1976; 4: 216219

2. Schwerdtfeger K, Jelasic F. Trismus in postoperative, posttraumatic and other brain stem lesions caused by paradoxical activity of masticatory muscles. Acta Neurochir 1985; 76: 62-66
3. Kadyan V, Clairmont AC, Engle M, Colachis SC. Severe trismus as a complication of cerebrovascular accident: a case report. Arch Phys Med Rehabil 2005; 86: 594-595

4. Fietzek UM, Kossmehl P, Barthels A, Ebersbach G, Zynda B, Wissel J. Botulinum toxin B increases mouth opening in patients with spastic trismus. Eur J Neurol 2009; 16: 1299-1304

5. Restivo DA, Maimone D, Patti F, Marchese-Ragona R, Marino G, Pavone A. Trismus after stroke/TBI: botulinum toxin benefit and use pre-PEG placement. Neurology 2005; 64: 2152-2153

6. Jelasic F, Freitag V. Inverse activity of masticatory muscles with and without trismus: a brainstem syndrome. J Neurol Neurosurg Psychiatry 1978; 41: 798-804

7. Beradelli A, Abbruzzese G, Bertolasi L, Cantarella G, Carella F, Curra A, Grandis D, DeFazio G, Galardi G, Girlanda $P$, et al. Guidelines for the therapeutic use of botulinum toxin in movement disorders. Italian study group for movement disorders, Italian Society of neurology. Ital J Neurol Sci 1997; 18: 261-269

8. Dressler D. Botulinum toxin for treatment of dystonia. Eur J Neurol 2010; 17 Suppl 1: 88-96

9. Ward AB. Spasticity treatment with botulinum toxins. J Neural Transm 2008; 115: 607-616

10. Spillane KS, Shelton JE, Hasty MF. Stroke-induced trismus in a pediatric patient: long-term resolution with botulinum toxin A. Am J Phys Med Rehabil 2003; 82: 485-488 